\title{
The highly ionized disk wind of GRO J1655-40
}

\author{
G. Sala ${ }^{1}$, J. Greiner ${ }^{1}$, J. Vink ${ }^{2}$, F. Haberl ${ }^{1}$, E. Kendziorra ${ }^{3}$, and X. L. Zhang ${ }^{1}$ \\ 1 Max-Planck-Institut für extraterrestrische Physik, Postfach 1312, 85741 Garching, Germany \\ e-mail: gsala@mpe.mpg.de \\ 2 Astronomical Institute, University Utrecht, PO Box 80000, 3508 TA Utrecht, The Netherlands \\ 3 Institut für Astronomie und Astrophysik, Abteilung Astronomie, Sand 1, 72076 Tübingen, Germany
}

Received 9 March 2006 / Accepted 31 August 2006

\section{ABSTRACT}

\begin{abstract}
Aims. The galactic superluminal microquasar GRO J1655-40 started a new outburst in February 2005, after seven years in quiescence, rising to a high/soft state in March 2005. In this paper we study the X-ray spectra during this rise.

Methods. We observed GRO J1655-40 with XMM-Newton on 27 February 2005 in the low/hard state, and on three consecutive days in March 2005 during the rise of the source to its high/soft state. The EPIC-pn camera was used in the fast-read burst mode to avoid photon pile-up.

Results. First, we contributed to the improvement of the calibration of the EPIC-pn, since the high flux received from the source required some refinements in the correction of the charge transfer efficiency of the camera. Second, we find that the X-ray spectrum of GRO J1655-40 is dominated in the high/soft state by the thermal emission from the accretion disk, with an inner radius of $13-14[\mathrm{D} / 3.2 \mathrm{kpc}] \mathrm{km}$ and a maximum temperature of $1.3 \mathrm{keV}$. We detected two absorption lines in the EPIC-pn spectra at 6.7-6.8 and 7.8-8.0 keV, which can be identified as the $\mathrm{K} \alpha$ and $\mathrm{K} \beta$ lines of either blended Fe XXV and Fe XXVI or blueshifted Fe XXV. We find no orbital dependence on the X-ray properties, which provides an upper limit for the inclination of the system of $73^{\circ}$. The RGS spectrometers reveal interstellar absorption features at 17.2 $\mathrm{A}, 17.5 \AA$ (Fe L edges), and $23.54 \AA$ (OI K $\alpha$ ). Finally, while checking the interstellar origin of the OI line, we found a general correlation of the OI K $\alpha$ line equivalent width with the hydrogen column density using several sources available in the literature.
\end{abstract}

Key words. X-rays: stars - stars: binaries: close - X-rays: individuals: GRO J1655-40

\section{Introduction}

Galactic microquasars are accreting binary systems that eject jets at relativistic velocities. Both black holes and neutron stars have been identified as the compact, accreting object. The analogy of these systems to extragalactic quasars and active galactic nuclei (AGN) make them excellent laboratories for studying the physics involved in accretion disks and the ejection of relativistic jets associated with accreting black holes (Mirabel et al. 1992). The physics ruling black hole systems is essentially the same in galactic microquasars, which host stellar black holes, and in AGN with supermassive black holes, but the differences in time scales makes the study of some aspects easier in microquasars than in AGN. The characteristic timescale for the flow of matter onto a black hole is proportional to its mass, being on the order of minutes in a microquasar of a few solar masses, but thousands of years in a massive black hole of $10^{9} M_{\odot}$. In addition, thanks to their proximity, microquasar jets have proper motions in the plane of the sky with velocities about a thousand times faster than AGN, and two-sided jets can be observed.

The microquasar GRO J1655-40 (X-ray nova Sco 1994, Zhang et al. 1994) was the second superluminal source discovered in our Galaxy after GRS 1915+105 (Mirabel \& Rodriguez 1994). The two sources may also be peculiar in the sense that both systems show evidence of containing a maximally spinning black hole (Zhang et al. 1997).

^ Based on observations obtained with XMM-Newton, an ESA science mission with instruments and contributions directly funded by ESA Member States and NASA.
Radio images of GRO J1655-40 showed twin jets with apparent superluminal motion (Tingay et al. 1995) moving in opposite directions at $0.92 \mathrm{c}$, and the distance was determined to be $3.2 \pm 0.2 \mathrm{kpc}$ (Hjellming \& Rupen 1995). Using the dust scattering halo observed by ROSAT, Greiner et al. (1995) determined a distance of $3 \mathrm{kpc}$, compatible with the previous determination. Optical observations in 1996 provided an inclination angle of $69.5 \pm 0.08^{\circ}$, a radial velocity semiamplitude of $228.2 \pm 2.2 \mathrm{~km} \mathrm{~s}^{-1}$, and a dynamical mass of the primary component of $7.02 \pm 0.22 M_{\odot}$ (Orosz \& Bailyn 1997), indicating that it is a black hole. Hubble Space Telescope observations showed that the system moves in an eccentric orbit with a runaway space velocity (i.e., with respect to the Galactic rotation corresponding to its position in the Galactic plane) of $112 \pm 18 \mathrm{~km} \mathrm{~s}^{-1}$ (Mirabel et al. 2002), which together with abundance anomalies found in the atmosphere of the donor star (Israelian et al. 1999) provide evidence of a supernova origin of the black hole in GRO J1655-40.

ASCA observations of GRO J1655-40 in August 1994 and August 1995 provided the first detection of absorption lines in an accretion powered source (Ueda et al. 1998). The energy of the lines was found to depend on the X-ray intensity, being $6.95 \mathrm{keV}(\mathrm{Fe} \mathrm{XXVI} \mathrm{K} \alpha)$ at $2.2 \mathrm{Crab}$ and 6.63 and $7.66 \mathrm{keV}$ (Fe XXV K $\alpha$ and $\mathrm{K} \beta$ ) at $0.27-0.57 \mathrm{Crab}$, revealing the presence of a highly ionized absorber. Similar absorption features were also detected for GRS 1915+105 (Kotani et al. 2000). During the outburst in 1997, ASCA observed GRO J1655-40 between 25 and 28 February, when it was at $1.1 \mathrm{Crab}$, and revealed an absorption feature at $6.8 \mathrm{keV}$, interpreted as the 


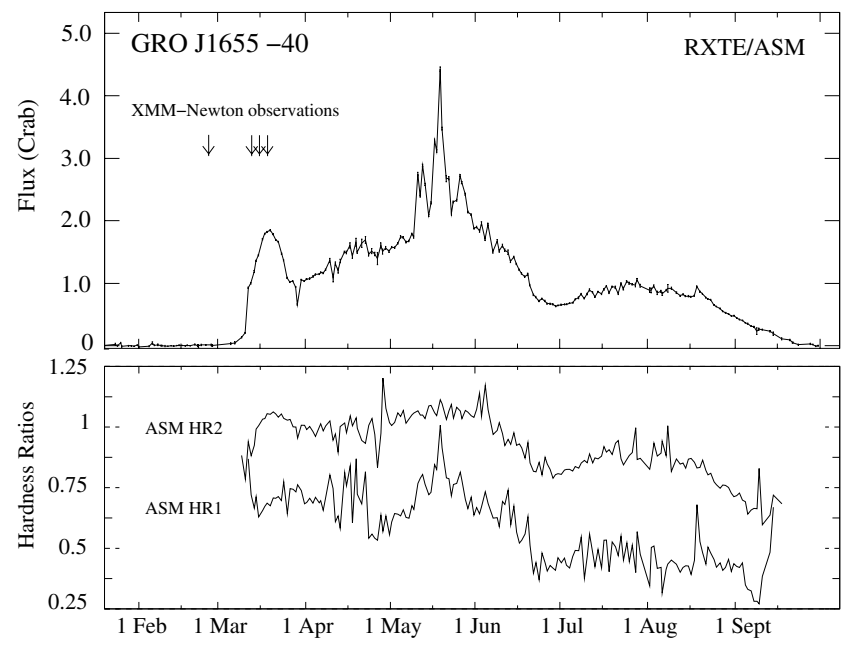

Fig. 1. RXTE/ASM light curve of GRO J1655-40, with dates of our XMM-Newton observations, and ASM hardness ratios: HR1, ratio of the ASM count rates $(3-5 \mathrm{keV}) /(1.5-3 \mathrm{keV})$, and HR2, (5$12 \mathrm{keV}) /(3-5 \mathrm{keV})$. Data from the quick-look results provided by the ASM/RXTE team.

blend of the two resonance $\mathrm{K} \alpha$ lines of Fe XXVI and Fe XXV (Yamaoka et al. 2001). Simultaneously, RXTE observations performed on 26 February 1997 showed emission lines at 5.85 and $7.32 \mathrm{keV}$, interpreted as the first red- and blueshifted Fe K $\alpha$ disk line in a Galactic source (Balucinska-Church \& Church 2000) and indicating the presence of significantly ionized material in a region of the disk at $\sim 10$ Schwarzschild radii.

The first observed X-ray outbursts of GRO J1655-40 occurred in 1994-1995 (Zhang et al. 1994; Harmon et al. 1995), and a 16-month-long outburst began on 25 April 1996 (Sobczak et al. 1999). After 7 years of inactivity, GRO J1655-40 left quiescence again on 17 February 2005 (Markwardt \& Swank 2005). The X-ray evolution was followed with RXTE/ASM (Homan et al. 2005) and Swift (Brocksopp et al. 2006). In February GRO J1655-40 entered a low/hard state (Homan 2005) until it experienced a first outburst in March, moving into a high/soft state and reaching $~ 2 \mathrm{Crab}$ (Fig. 1). The decay of the first outburst was followed by a month and a half of increasing X-ray flux and finally a strong outburst in a very high state in May 2005, when the source reached more than $4 \mathrm{Crab}$.

Here we present XMM-Newton observations performed in February-March 2005, aimed at obtaining detailed spectroscopy during the rise to the high/soft state. We use the canonical distance of $3.2 \mathrm{kpc}$ throughout (see note added in proof, however).

\section{Observations and data reduction}

We observed GRO J1655-40 with XMM-Newton some days before the rise to the soft/high state on 27 February 2005 (40 ks; obs. id. 0112921-301) and again on 14, 15, and 16 March 2005, close to the maximum of the first outburst (15 ks each, obs. id. 0112921-401/-502/-601; see Fig. 1 and Table 1). The EPIC-pn camera was used in the burst mode, a fast read-out mode that permits the observation of very bright sources with a high time resolution (Kuster et al. 1999). In the burst mode, only one of the 12 CCDs of the camera is used. The electrons released in the area of the CCD where the source is positioned are quickly shifted towards the read-out node to prevent pile-up and then normally read out. In this mode, the spatial information in one dimension (shift direction) is lost and only $3 \%$ of the total time is effectively used, but bright sources can be observed without photon pile-up problems. The two MOS cameras were switched off for telemetry limitations, while the RGS spectrometers were operating in normal spectroscopy mode.

The XMM-Newton data were reduced using SAS 6.1, and XSPEC 11.3 was used for spectral analysis. Solar flares partially affected our observations, and some time intervals could not be used for analysis (in particular, the second half of the first observation lasting $20 \mathrm{ks}$, the first $2 \mathrm{ks}$ of the second and the first $5 \mathrm{ks}$ of our last observation). For the EPIC-pn data, only single events with energy higher than $0.4 \mathrm{keV}$ were taken into account. Events with RAWY $\geq 140$ were excluded to avoid direct illumination by the source, and the source spectra were extracted from a box including 10 RAWX columns at each side of the source position and also ignoring RAWY $\leq 10$. The high luminosity of GRO J1655-40 at the time of our XMM-Newton observations (almost twice as bright as the Crab, the source used for the burst mode calibration) made evident a rate-dependence of the charge transfer efficiency (CTE) that was not included in the calibration. We determined this dependence and corrected our spectra accordingly (see Appendix).

In the RGS spectra, the energy range $0.3-2.0 \mathrm{keV}$ was initially included. We applied a preliminary version of the wavelength and time-dependent corrections to the RGS effective area, which will be implemented in the next release of the XMM-Newton software. Nevertheless, during our three March observations, strong differences were found between the responses of the different nodes of the RGS, probably due to the high flux of the source. We have thus ignored the energy ranges with evident problems, more precisely, energies above $1 \mathrm{keV}$ for the first order in all cases and between 0.85 and $1.2 \mathrm{keV}$ in the second order of the RGS2 on the 14 March observation. Since both first and second order spectra of the two RGS are available, no energy range remains uncovered despite the rejection of these data.

Simultaneous RXTE observations of GRO J1655-40 are publicly available (Table 1). We have included the standard spectral products from the PCA and HEXTE instruments in our analysis, to constrain the model components at hard X-rays. When attemps are made to fit EPIC-pn and PCA spectra of March observations simultaneously, large residuals appear in the PCA spectrum for energies lower than $\sim 6 \mathrm{keV}$, in the region of the instrumental Xenon edges. Since the accuracy of the PCA response function is limited by the poor modeling of these edges (Jahoda et al. 2006), it is possible that the high flux of the source in these observations is revealing some inaccuracy in the calibration, as in the case of the XMM-Newton instruments. We thus only consider the PCA spectrum for energies above $6 \mathrm{keV}$.

Neither orbital variations, like eclipses or periodic modulations, nor dips are observed in the light curve, and the flux of the source remains constant within $30 \%$.

\section{Spectral analysis}

During the first observation on 27 February 2005, before the start of the first outburst, the source was in a low/hard state, with the XMM-Newton plus RXTE instruments showing a spectrum dominated by an absorbed power law with photon index $\Gamma=1.48 \pm 0.01$ (uncorrelated $90 \%$ error for 1 degree of freedom). This agrees with the results from RXTE observations starting only two days later, in the period 1-6 March 2005 ( $\Gamma=1.4-$ 1.6, Swank \& Markwardt 2005), and about $10 \%$ flatter than reported by Brocksopp et al. (2006) from Swift observations on 
Table 1. Observation log.

\begin{tabular}{ccccccc}
\hline \hline Instrument & Date & $\begin{array}{c}\text { Start } \\
(\mathrm{UTC})\end{array}$ & $\begin{array}{c}\text { End } \\
(\mathrm{UTC})\end{array}$ & $\begin{array}{c}\text { Exposure } \\
(\mathrm{ks})\end{array}$ & $\begin{array}{c}\text { Energy range } \\
(\mathrm{keV})\end{array}$ & $\begin{array}{c}\text { Flux }\left(\times 10^{-10}\right) \\
\left(\mathrm{erg} \mathrm{s}^{-1} \mathrm{~cm}^{-2}\right)\end{array}$ \\
\hline XMM/EPIC-pn & $27-02-2005$ & $07: 56$ & $19: 34$ & 41.8 & $0.4-10.0$ & 2.5 \\
XMM/RGS1 \& 2, order 1 & $27-02-2005$ & $07: 46$ & $19: 35$ & 42.5 & $0.3-2.0$ & 0.5 \\
XMM/RGS1 \& 2, order 2 & $27-02-2005$ & $07: 46$ & $19: 35$ & 42.5 & $0.54-2.0$ & 0.4 \\
RXTE/PCA & $27 / 28-02-2005$ & $20: 37$ & $02: 53$ & 13.0 & $6.0-25.0$ & 5.9 \\
RXTE/HEXTE & $27 / 28-02-2005$ & $20: 37$ & $02: 17$ & 4.2 & $20-200$ & 21 \\
XMM/EPIC-pn & $14-03-2005$ & $16: 27$ & $20: 36$ & 14.9 & $0.4-10.0$ & 214 \\
XMM/RGS1 \& 2, order 1 & $14-03-2005$ & $16: 17$ & $20: 37$ & 15.6 & $0.3-1.0$ & 2.9 \\
XMM/RGS1 \& 2, order 2 & $14-03-2005$ & $16: 17$ & $20: 37$ & 15.6 & $0.48-1.8$ & 40 \\
RXTE/PCA & $14-03-2005$ & $13: 02$ & $19: 17$ & 12.9 & $6.0-25.0$ & 54 \\
RXTE/HEXTE & $14-03-2005$ & $13: 02$ & $19: 17$ & 4.0 & $20-200$ & 36 \\
XMM/EPIC-pn & $15-03-2005$ & $16: 27$ & $20: 36$ & 14.9 & $0.4-10.0$ & 208 \\
XMM/RGS1 \& 2, order 1 & $15-03-2005$ & $16: 17$ & $20: 37$ & 15.6 & $0.3-1.0$ & 2.9 \\
XMM/RGS1 \& 2, order 2 & $15-03-2005$ & $16: 17$ & $20: 37$ & 15.6 & $0.48-1.8$ & 40 \\
RXTE/PCA & $15-03-2005$ & $14: 08$ & $19: 26$ & 11.6 & $6.0-25.0$ & 48 \\
RXTE/HEXTE & $15-03-2005$ & $14: 08$ & $19: 36$ & 3.5 & $20-200$ & 16 \\
XMM/EPIC-pn & $16-03-2005$ & $16: 14$ & $20: 23$ & 14.9 & $0.4-10.0$ & 260 \\
XMM/RGS1 \& 2, order 1 & $16-03-2005$ & $16: 04$ & $20: 24$ & 15.6 & $0.3-1.0$ & 3.2 \\
XMM/RGS1 \& 2, order 2 & $16-03-2005$ & $16: 04$ & $20: 24$ & 15.6 & $0.54-1.8$ & 45 \\
RXTE/PCA & $16-03-2005$ & $17: 08$ & $22: 37$ & 11.1 & $6.0-25.0$ & 70 \\
RXTE/HEXTE & $16-03-2005$ & $17: 08$ & $22: 37$ & 3.5 & $20-200$ & 34 \\
\hline
\end{tabular}

Table 2. Continuum spectral model for XMM-Newton and RXTE observations.

\begin{tabular}{|c|c|c|c|c|}
\hline Parameters & 27 Feb. 2005 & 14 March 2005 & 15 March 2005 & 16 March 2005 \\
\hline$N_{\mathrm{H}}\left(\times 10^{21}\right) \mathrm{cm}^{-2}$ & $5.2 \pm 0.2$ & $5.46 \pm 0.02$ & $5.57 \pm 0.02$ & $5.45 \pm 0.03$ \\
\hline Dust fraction ${ }^{a}$ & $0.15 \pm 0.03$ & $0.186 \pm 0.002$ & $0.169 \pm 0.003$ & $0.184 \pm 0.003$ \\
\hline$k T_{\text {in }}(\mathrm{keV})$ & - & $1.231 \pm 0.003$ & $1.268 \pm 0.003$ & $1.326 \pm 0.003$ \\
\hline$R_{\text {in }}^{b}(\mathrm{~km})$ & - & $14.25 \pm 0.05$ & $13.25 \pm 0.05$ & $13.55 \pm 0.05$ \\
\hline$\Gamma$ & $1.48 \pm 0.01$ & $2.10 \pm 0.01$ & $2.27 \pm 0.01$ & $2.13 \pm 0.01$ \\
\hline$K^{c}\left(\mathrm{ph} \mathrm{keV}^{-1} \mathrm{~cm}^{-2} \mathrm{~s}^{-1} @ 1 \mathrm{keV}\right)$ & $0.0385 \pm 0.0007$ & $0.94 \pm 0.02$ & $0.76 \pm 0.07$ & $1.05 \pm 0.02$ \\
\hline$L_{0.4-10 \mathrm{keV}}^{d}\left(\mathrm{erg} \mathrm{s}^{-1}\right)$ & $3.7 \times 10^{35}$ & $3.4 \times 10^{37}$ & $3.4 \times 10^{37}$ & $4.2 \times 10^{37}$ \\
\hline
\end{tabular}

${ }^{a}$ Dust scattering fraction at $1 \mathrm{keV}$. Halo size is fixed to 10 times the detector beam-size at $1 \mathrm{keV} .{ }^{b}$ For a distance of $3.2 \mathrm{kpc}$ and an inclination of $70^{\circ} .{ }^{c}$ Normalization constant for the power law component. ${ }^{d}$ Luminosity in the range $0.4-10 \mathrm{keV}$, for a distance of $3.2 \mathrm{kpc}$.

Table 3. Features in RXTE/PCA spectra.

\begin{tabular}{ccccc}
\hline \hline Parameters & 27 February 2005 & 14 March 2005 & 15 March 2005 & 16 March 2005 \\
\hline Line energy (keV) (FIXED) & 6.4 & - & - & - \\
$1 \sigma$ line width (eV) & $300 \pm 100$ & - & - & - \\
Equivalent width (eV) & $135 \pm 20$ & - & - & - \\
Identification & Fe I & - & - & - \\
Edge energy $(\mathrm{keV})$ & - & $8.68 \pm 0.05$ & $8.57 \pm 0.03$ & $8.77 \pm 0.04$ \\
Edge optical depth & - & $0.44 \pm 0.05$ & $0.5 \pm 0.2$ & $0.57 \pm 0.05$ \\
Edge width & - & $1.2 \pm 0.2$ & $1.9 \pm 0.5$ & $1.3 \pm 0.2$ \\
\hline
\end{tabular}

6 March $2005(\Gamma=1.72 \pm 0.03)$. The fit with a power law leaves excess residuals at low energies both in the EPIC-pn and the RGS spectra, but the fit improves when the spectrum is modified by the scattering of dust in the line-of-sight. In ROSAT HRI observations in 1994, Greiner et al. (1995) found a halo around GRO J1655-40 due to the scattering of the X-rays by interstellar dust. We thus modify our spectral model with the dust model available in XSPEC, which assumes that the scattered flux appears as a uniform disk on the sky (as for a source that is constant in time) whose size has a $1 / E$ dependence and whose total flux has a $1 / E^{2}$ dependence.
The RXTE/PCA spectrum also shows residuals between 6-7 keV, which are well-fitted with an iron fluorescence line with energy fixed at $6.4 \mathrm{keV}$, for which we derive an equivalent width of $135 \pm 20 \mathrm{eV}$. The line is not seen in the EPICpn data, and by fixing the energy at $6.4 \mathrm{keV}$, we obtain an upper limit of $140 \mathrm{eV}$ for the equivalent width. The presence of an iron fluorescence line in the low/hard state is confirmed by Swift observations one week after our XMM-Newton observation (Brocksopp et al. 2006). All parameters of the fit for this first observation are listed in Tables 2 and 3. 


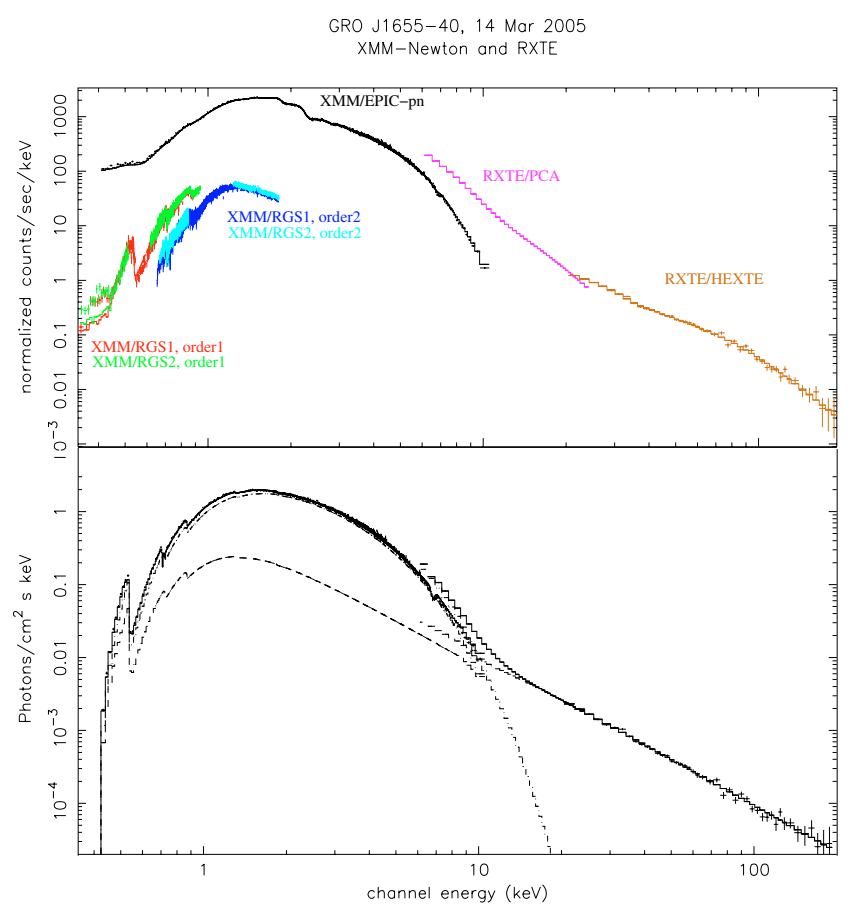

Fig. 2. XMM-Newton and RXTE observed spectra (upper panel) and unfolded spectra showing the contribution of the thermal disk emission (soft energies) and the power law (hard energies) to the total spectrum (lower panel). Residuals for the three March observations are shown in Figs. 3-5.

The three March observations have very similar spectra, with the XMM-Newton continuum spectra dominated by an absorbed multicolor disk (MCD), where $k T_{\text {in }}$ increases with time from 1.25 to $1.35 \mathrm{keV}$, and the higher energy spectra from RXTE represented by a soft power law, with $\Gamma=2.1-2.3$ (see Fig. 2 and Table 2). The simultaneous fit of the EPIC-pn, RGS, PCA, and HEXTE data with the MCD-plus-power-law model leaves large residuals again below $0.6 \mathrm{keV}$ in both the EPICpn and the RGS spectra, as well as in the range $8-15 \mathrm{keV}$ in the XTE/PCA spectra.

The soft excess in the XMM-Newton instruments, as in the case of the February observation, is most probably related to the dust scattering. Although the global fit improves after adding the dust's halo effect, the RGS spectra still show an excess for energies lower than $0.5 \mathrm{keV}$. Using different abundance tables or leaving the abundances as free parameters did not help for fitting this soft excess. This is probably related to the more limited sky area included in the RGS than in the EPIC-pn extraction regions. A detailed analysis of the halo, including spatially resolved spectroscopy, is currently underway and will be reported separately. As a first approximation, fixing the dust halo size to 10 times the beam size, from the fit with the EPIC-pn data, we obtain in all cases a scattering fraction at $1 \mathrm{keV}$ of $17-20 \%$, similar to the $21 \%$ relative intensity of the halo found by Greiner et al. (1995).

The quality of the fit in the $8-15 \mathrm{keV}$ energy range spectra of the RXTE instruments during the three March observations improves with the addition of a smeared edge at $8.6-8.8 \mathrm{keV}$ (see Table 3). An iron edge at $8.81 \mathrm{keV}$ was also detected in the 1994 outburst from ASCA observations (Ueda et al. 1998) and at $8 \mathrm{keV}$ with RXTE observations during the 1996-1997 outburst (Sobczak et al. 1999). Nevertheless, for the 15 and 16 March observations, even after including the edge, the fit of the PCA spectra below $20 \mathrm{keV}$ is poor. It is worth noticing that

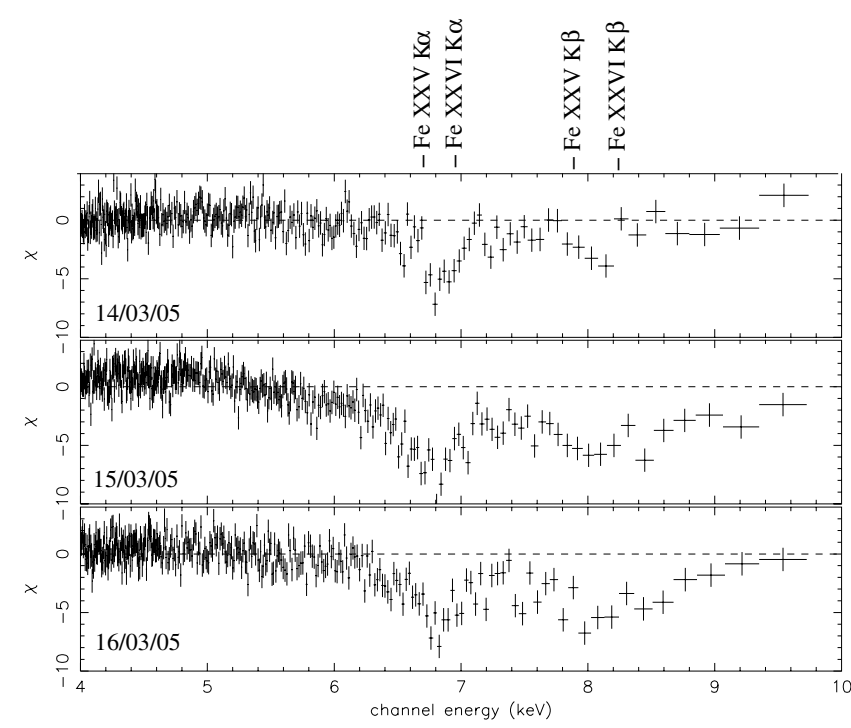

Fig. 3. EPIC pn residuals of GRO J1655-40 spectra of our three March 2005 observations at zero velocity, after fitting an absorbed multitemperature disk model.

Sobczak et al. (1999) also did not find a good fit for the PCA spectra between 2.5 and $20 \mathrm{keV}$ with the MCD-plus-power-law model, whether including emission lines, edges, or other effects, for the observations performed during the rise of the source to the first burst in 1996, in a phase of the light curve similar to our March observations.

Clear structured residuals also appear in the EPIC-pn spectrum at $\sim 6.8$ and $\sim 7.9 \mathrm{keV}$, corresponding to absorption features in the Fe K region (see Fig. 3 and Table 4). In all three March observations, the energy of the lines is the same, corresponding to the $\mathrm{K} \alpha$ and $\mathrm{K} \beta$ lines of either blended Fe XXV and Fe XXVI, or blueshifted Fe XXV. In the latter case, given the energy resolution of the EPIC-pn $(150 \mathrm{eV} @ 6.4 \mathrm{keV})$, the velocity is not well-constrained.

In the three March observations, the fit of the RGS spectra around the interstellar neutral oxygen edge at 22.6-23.2 (534-549 eV) with the phabs absorption model is unacceptable unless the "Vern" cross-sections (Verner et al. 1996) available in XSPEC are used. Even in this case, the fit leaves clear residuals, indicating the presence of an additional absorption line at $23.5 \AA$ (Fig. 4), which corresponds to the $1 \mathrm{~s}-2 \mathrm{p}$ absorption line of neutral oxygen. Given the energy resolution of the RGS, the observed maximum width of the line is compatible with it being a narrow line originating in the interstellar medium. The RGS spectra also show clear absorption features at 17.2 $\AA$ and $17.5 \AA$ (Fig. 5), which correspond to Fe $\mathrm{L}_{2,3}\left(2 \mathrm{p}_{1 / 2}\right.$, $2 \mathrm{p}_{3 / 2}$ ) edges, also observed in the interstellar absorption towards some other sources (Wilms et al. 2000; Paerels et al. 2001; Schulz et al. 2002). As in these previous cases, simple edge models do not provide a good fit for these features.

Since both the line at $23.5 \AA$ and the edges at $17.2 \AA$ and $17.5 \AA$ are caused by interstellar gas, but are not reproduced well by the simple phabs model in XSPEC, the recent new version of the TBabs model (Wilms et al. 2006, http://astro. uni-tuebingen.de/ wilms/research/tbabs/) has been used. This has been shown to provide a good fit to the complex oxygen edge (Juett et al. 2004), including two edges and five absorption lines, and to the iron absorption features (Juett et al. 2006). It also provides a good fit for our RGS features (see Figs. 4 and 5), with a hydrogen column 


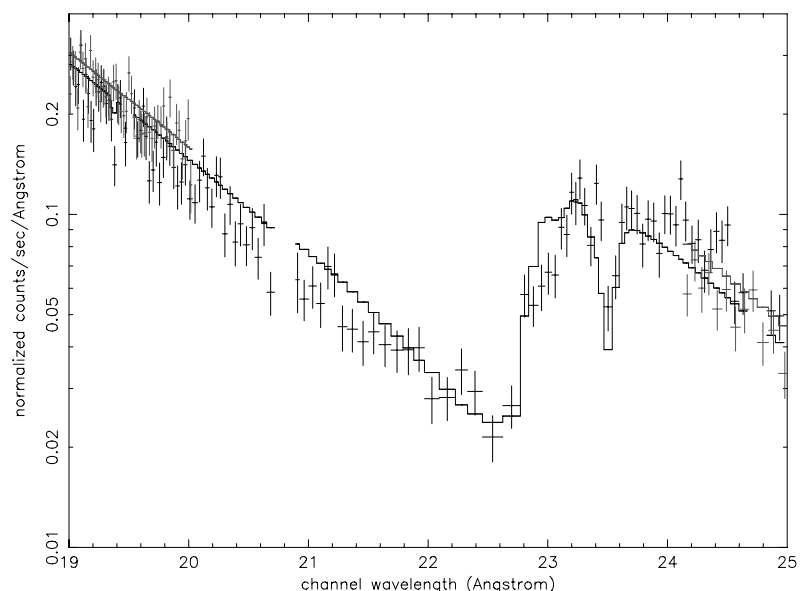

Fig. 4. RGS1 (black) and RGS2 (grey, contributing only below $20 \AA$ and above $24 \AA$ ) around the oxygen edge in the first-order spectra of the 14 March 2005 observation, fitted with the new TBabs model.

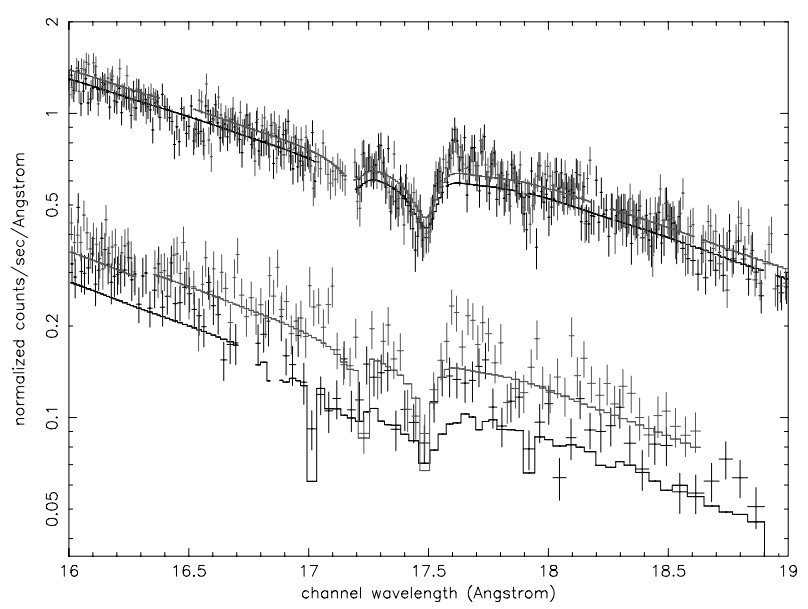

Fig. 5. First and second order (with lower count rate) spectra of GRO J1655-40 in the range 16-19 $\AA$, obtained with RGS1 (black) and RGS2 (grey) on 14 March 2005, showing the ISM Fe L edges fitted with the new TBabs model.

$N_{\mathrm{H}}: 7.3( \pm 0.3), \quad 7.0( \pm 0.3), \quad 6.9( \pm 0.5) \times 10^{21} \mathrm{~cm}^{-2}, \quad$ and an overabundance of oxygen with respect to solar of $1.45( \pm 0.15), 1.6( \pm 0.1), 1.45( \pm 0.15)$ (for days 14,15 , and 16 March 2005, respectively).

\section{Discussion}

The spectrum of GRO J1655-40 during our March 2005 observations is dominated by the thermal emission of the accretion disk plus a power law component at high energies. The soft X-ray spectrum is affected by dust scattering and interstellar absorption, while absorption lines of highly ionized species of Fe are observed in the EPIC-pn spectrum. In the following sections, we discuss the physical interpretation of each of these components separately. We also discuss the constrains on the inclination of the system derived from our observations.

\subsection{The dust halo}

The effect of the scattering dust halo is clearly detected in the soft X-ray spectra. The scattering fraction at $1 \mathrm{keV}$ is changing during the three March observations, decreasing from
$18.6( \pm 0.2) \%$ to $16.9( \pm 0.3) \%$ in the first $24 \mathrm{~h}$ and then increasing again to $18.4( \pm 0.3) \%$ during the next day. This temporal variation is consistent with a constant dust halo but a variable X-ray source flux: the time needed for the soft photons originating on the disk to travel to the scattering site far away from the source causes a delay in the flux variations of the dust halo with respect to the variations of the source. As a result, just after an increase in the disk temperature and thus in the source's soft spectrum, the fraction of scattered photons with respect to the source is temporally smaller (during the second March observation) until the increasing source photons reach the outer part of the halo and their scattered photons also reach the observer, again increasing the scattering fraction (third March observation). This effect has been known for some time. It was also observed by ROSAT and used to estimate the distance to GRO J1655-40 (Greiner et al. 1995).

\subsection{Continuum}

The maximum disk temperature observed in the March observations, increasing from 1.25 to $1.35 \mathrm{keV}$, is consistent with the values commonly observed in blackhole binaries in the high/soft state (see for instance McClintock \& Remillard 2006) and with the Swift observations of the same period (Brocksopp et al. 2006). The inner radius, $R_{\text {in }} \sim 13-14[D / 3.2 \mathrm{kpc}] \mathrm{km}$, is only a little larger than the gravitational radius of the black hole, $R_{\mathrm{g}}=G M / c^{2}=10 \mathrm{~km}$, consistent with a rapidly spinning black hole (Zhang et al. 1997).

From the fit parameters of the MCD model, the luminosity of the disk can be calculated as $L_{\mathrm{x}}=4 \pi R_{\mathrm{in}}^{2} \sigma T_{\text {in }}^{4}$ (Makishima et al. 1986). With our values, the accretion disk luminosity is $6-7 \times 10^{37}[D / 3.2 \mathrm{kpc}]^{2} \mathrm{erg} \mathrm{s}^{-1}$.

With the accretion disk luminosity, the accretion rate can be calculated as $2 L_{\mathrm{x}} R_{\text {in }} / G M g^{2}$ (Makishima et al. 1986), where $g=$ $\left(1-R_{\mathrm{g}} / R_{\mathrm{in}}\right)^{1 / 2}$ is a correction for the general relativity, $R_{\mathrm{g}}$ the gravitational radius, and $M$ the mass of the black hole. From the accretion-disk luminosity, the disk inner radius, and the blackhole mass of GRO J1655-40, we obtain an accretion rate during the high/soft state of $\sim 10^{-8} M_{\odot} \mathrm{yr}^{-1}$.

The rise of the disk blackbody component during the high/soft state in March is reflected in the power law index of the corona, which increases from $1.48 \pm 0.01$ in February 27 to values higher than 2 in the March observations. With the rise in the thermal disk emission, the soft luminosity increases and the electrons in the corona are cooled more efficiently, resulting in a softer spectrum for the comptonized photons, i.e., a steeper power law.

\subsection{Interstellar absorption}

The interstellar OI K $\alpha$ line is clearly detected at $23.5 \AA$ in our three March 2005 RGS spectra. This line was also found in the RGS spectra of other sources and distinguished from the instrumental components around the interstellar oxygen edge, at 23.05 and $23.35 \AA$ (de Vries et al. 2003). Juett et al. (2004) detected the same line in the high resolution spectra of seven X-ray binaries using the Chandra/HETGS, as part of their study of the structure of the oxygen absorption edge caused by the interstellar medium. More recently, it was also detected in the Cyg X-2 spectrum (Costantini et al. 2005) in the observations that allowed the first spatially-resolved spectroscopic study of a scattering dust halo, as well as in the absorption towards LMC X-3 
(Wang et al. 2005) and the recently discovered black hole candidate XTE J1817-330 (Sala \& Greiner 2006).

To check the interstellar origin of the OI K $\alpha$ line observed in the RGS spectra of GRO J1655-40, we looked for a correlation of the equivalent width of the line and the hydrogen column density, using the above-mentioned works (Fig. 6). We find that the equivalent width of the $\mathrm{OI} \mathrm{K} \alpha$ line, $E W$, is indeed correlated with the column density, $N_{\mathrm{H}}$, as $E W(\mathrm{eV})=0.5( \pm 0.1)+2.8( \pm 0.3) \times$ $10^{-22} N_{\mathrm{H}}\left(\mathrm{cm}^{-2}\right)$. The values observed for GRO J1655-40 fall well within this correlation for the ISM. We find that an alternative correlation with a zero regression constant could be $E W(\mathrm{eV})=3.6( \pm 1.6) \times 10^{-22} N_{\mathrm{H}}\left(\mathrm{cm}^{-2}\right)$, but this would underestimate the equivalent width for the sources with hydrogen column density less than $5 \times 10^{21} \mathrm{~cm}^{-2}$.

\subsection{Orbital dependence and inclination of the binary system}

Since the orbital period of GRO J1655-40 is $~ 2.6$ days and our three March observations were taken in one day intervals, we cover approximately one whole orbital cycle. We can extrapolate the Orosz \& Bailyn (1997) ephemeris to obtain the orbital phase of our observations (error is 0.08 in all cases): $0.31-0.40$ (27 February), 0.17-0.24 (14 March), 0.55-0.62 (15 March), and $0.93-0.00$ (16 March).

Given the inclination of the system (between $\sim 70^{\circ}$, Orosz \& Bailyn 1997, van der Hooft et al. 1998; and $\sim 85^{\circ}$, Hjellming \& Rupen 1995), an orbital phase close to zero corresponds to having the donor star situated closer to the observer, while the disk would be in front of the secondary star in phase 0.5 . This means that if any of the observed spectral features were arising from the illuminated face of the secondary star, it would have its maximum at phase 0.5 and would not be present at phase zero. This is not the case in our observations. Another possible orbital effect could be that the disk emission were absorbed by the stellar wind of the secondary star, which would produce increased absorptions close to phase zero, i.e. on 16 March, which is also not the case.

Finally, neither dips nor eclipses were observed in the disk thermal emission at any of the phases. Given the size of the donor star, $\sim 5 R_{\odot}$ (Orosz \& Bailyn 1997), the binary separation $\left(1.17 \times 10^{12} \mathrm{~cm}\right)$, and assuming that the soft X-ray emission originates in the central $200000 \mathrm{~km}$ of the disk (see below, Sect. 4.5), the duration of a possible eclipse of the soft X-ray emission by the donor star would last more than $2.5 \mathrm{~h}$ (i.e., a change in the orbital phase of 0.04 ) close to phase zero. This should have been clearly visible during our 16 March observation.

The fact that no orbital modulation was observed in the $\mathrm{X}$-ray spectra provides an upper limit for the inclination of the system. With the parameters mentioned above for the sizes of the system, the inclination must be smaller than $73^{\circ}$ (for the innermost $200000 \mathrm{~km}$ of the disk surface to be visible in all orbital phases). This limit is in agreement with the inclination determined from the optical light-curve by Orosz \& Bailyn (1997), $69^{\circ} .5 \pm 0.8$, and by van der Hooft et al. (1998), 67.2 \pm 3.5 , but incompatible with the inclination inferred from the radio jets, $\sim 84^{\circ}$ (Hjellming \& Rupen 1995). This points, as suggested by Orosz \& Bailyn (1997), to an inclination of the jet axis of about $15^{\circ}$ with respect to the normal to the orbital plane. Alternatively, non-symmetric jet ejections could have lead to a wrong inclination inference (Hjellming \& Rupen 1995).

\subsection{The Fe $X X V / F e X X V I$ absorber}

Clear highly ionized $\mathrm{Fe}$ absorption lines were detected in the EPIC-pn spectra of the three March observations in the high/soft state. It is worth noting that Ueda et al. (1998) simultaneously found Fe XXV absorption features and an iron edge, while they detected no edge when absorption in the iron $K$ band corresponded to Fe XXVI.

Assuming that the absorbing plasma is photoionized, the ionization state of the observed elements provides information on the conditions and location of the absorber. The presence of Helike $\mathrm{Fe}$ ions indicates an ionization parameter $\xi=L / n r^{2} \sim$ $10^{3} \mathrm{erg} \mathrm{cm} \mathrm{s}^{-1}$ (Kallman et al. 1996). The lack of lower ionization absorption implies that the absorber cannot cover a large extent.

The equivalent width of the Fe lines increases between the first and the last March observations. This may be pointing out an increase either in the total column density or in the ionization state of the gas.

Ueda et al. (1998) determined the iron column density of the plasma to be $10^{19}-10^{20} \mathrm{~cm}^{-2}$ from the observed equivalent width and the curve of growth of the $\mathrm{Fe} X X V \mathrm{~K} \alpha$ line, which relates the expected equivalent width to the iron column density. Using their curve of growth and assuming the detected features are only Fe XXV, our equivalent width of the Fe XXV K $\alpha$ line (between 50 and $160 \mathrm{eV}$ ) corresponds to a column density of $10^{19}$ and $5 \times 10^{20} \mathrm{~cm}^{-2}$, which is similar to the values found by Ueda et al. (1998). Assuming cosmic abundances, this corresponds to a hydrogen column density in the range $2 \times 10^{23}-10^{25} \mathrm{~cm}^{-2}$. Since the observed absorption lines may be a blend of Fe XXV and Fe XXVI, these must be taken as upper limits. In addition, for a hydrogen column density higher than $10^{24} \mathrm{~cm}^{-2}$, the line absorber would be optically thick to Thomson scattering (Ueda et al. 1998). We consider this to be unlikely and, taking into account the errors in the equivalent widths, the column density could then be in the range $2 \times 10^{23}-10^{24} \mathrm{~cm}^{-2}$ in all three observations.

With the photoionization parameter, $\xi \sim 10^{3} \mathrm{~cm} \mathrm{~s}^{-1}$, and the flux detected above $\sim 9 \mathrm{keV}$ (X-rays photoionizing He-like iron ions), $L_{\geq 9 \mathrm{keV}} \sim 5 \times 10^{36} \mathrm{erg} \mathrm{s}^{-1}$ (for a distance of $3.2 \mathrm{kpc}$ ), the column density indicates a distance to the central source between 50000 and $200000 \mathrm{~km}$. Assuming that the disk radius is $70 \%$ of the Roche lobe radius, i.e., $4 \times 10^{6} \mathrm{~km}$, the Fe XXV absorber extends to less than $5 \%$ of the disk surface.

In the case where the features correspond to blueshifted Fe XXV lines, the error in the energy determination is too large to reach any strong conclusion about its blueshift. Nevertheless, assuming the wind velocity is constant, the common range of blueshifts of the three March observations is reduced to 2600-4500 $\mathrm{km} \mathrm{s}^{-1}$. From the parameters derived above for the Fe XXV absorber, and assuming constant density and spherical symmetry for the expanding wind, the mass loss rate $4 \pi r^{2} \rho v b$ would be in the range $(2-13) \times 10^{-7} b M_{\odot} \mathrm{yr}^{-1}$, with $b$ the filling factor. This upper limit is a factor 20-130 larger than the accretion rate, which would indicate a highly non-stationary situation. However, there is considerable uncertainty in the wind mass loss, since both the wind density and wind velocity are likely to be a function of radius, and the wind may be conical rather than spherically symmetric. Exploring these effects, however, goes beyond the scope of this paper.

\section{Summary}

GRO J1655-40 was observed with XMM-Newton during the low/hard state in February 2005 and in the soft/high state in March 2005. The continuum spectrum is dominated by an absorbed power law in the low/hard state and by a multicolor disk blackbody in the soft/high state, with the maximum temperature 
Table 4. Absorption features in EPIC-pn spectra.

\begin{tabular}{cccc}
\hline \hline Parameters & 14 March 2005 & 15 March 2005 & 16 March 2005 \\
\hline Line energy $(\mathrm{keV})$ & $6.82 \pm 0.06$ & $6.71 \pm 0.06$ & $6.75 \pm 0.05$ \\
$1 \sigma$ line width $(\mathrm{eV})$ & $110_{-50}^{+150}$ & $200 \pm 100$ & $250 \pm 50$ \\
Equivalent width $(\mathrm{eV})$ & $60_{-10}^{+30}$ & $100 \pm 60$ & $110 \pm 30$ \\
Identification $^{a}$ & $\mathrm{Fe} \mathrm{XXV} \mathrm{K \alpha}\left(E_{0} 6.700 \mathrm{keV}\right) / \mathrm{Fe} \mathrm{XXVI} \mathrm{K \alpha}\left(E_{0} 6.952 / 6.973 \mathrm{keV}\right)$ \\
$v\left(\mathrm{~km} \mathrm{~s}^{-1}\right)^{b}$ & $-5200 \pm 2600$ & $-400 \pm 2700$ & $-2200 \pm 2200$ \\
Line energy $(\mathrm{keV})$ & $8.04 \pm 0.06$ & $7.85 \pm 0.15$ & $7.9 \pm 0.1$ \\
$1 \sigma$ line width $(\mathrm{eV})$ & $100_{-50}^{+100}$ & $700 \pm 200$ & $600 \pm 200$ \\
Equivalent width $(\mathrm{eV})^{a}$ & $54_{-25}^{+1}$ & $230_{-50}^{+20}$ & $240 \pm 40$ \\
Identification $^{a}$ & $\mathrm{Fe} \mathrm{XXV} \mathrm{K} \beta\left(E_{0}\right.$ & $7.872 / 7.881 \mathrm{keV}) / \mathrm{Fe} \mathrm{XXVI} \mathrm{K} \beta\left(E_{0} 8.246 / 8.252 \mathrm{keV}\right)$ \\
$v\left(\mathrm{~km} \mathrm{~s}^{-1}\right)^{b}$ & $-5900 \pm 2200$ & $1200 \pm 5700$ & $-700 \pm 3800$ \\
\hline
\end{tabular}

${ }^{a}$ Based on CHIANTI data base (Dere et al. 1997; Landi et al. 2006); ${ }^{b}$ Blueshift with respect to Fe XXV lines.

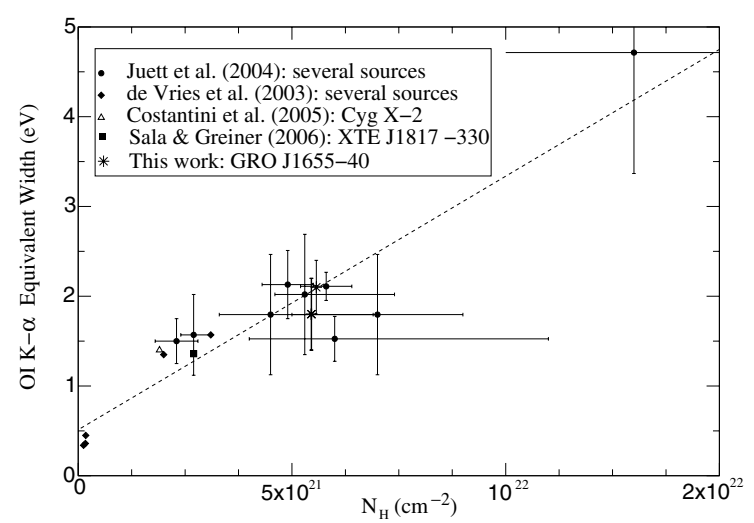

Fig. 6. OI K- $\alpha$ line equivalent width versus hydrogen column density for several galactic sources. The dashed line indicates an approximate linear correlation, $E W(\mathrm{eV})=0.5+2.8 \times 10^{-22} N_{\mathrm{H}}\left(\mathrm{cm}^{-2}\right)$.

increasing during the high state. In addition, the X-ray spectrum during the March observations shows several spectral features:

- Interstellar neutral oxygen causes an OI $\mathrm{K} \alpha$ absorption line at $23.5 \AA$. A correlation between the equivalent width of this line and the hydrogen column density has been obtained from published observations of several galactic X-ray sources, and the comparison of our observations with that correlation confirms the interstellar origin of the OI $\mathrm{K} \alpha$ absorption line.

- Fe XXV, possibly blended with Fe XXVI, is detected in absorption in the EPIC-pn spectra, indicating the presence of a highly ionized absorber with an ionization parameter of 1000 erg $\mathrm{cm} \mathrm{s}^{-1}$, extending less than $200000 \mathrm{~km}$ from the central source, and corresponding to $5 \%$ of the accretion disk.

Photoionized absorbers are seen in AGN as well, also with expansion velocities. The detection of photoionized winds in microquasars is then another similarity between these two kinds of systems. Nevertheless, winds in AGN are not uniform, but distributed instead in clouds of material. If this were the case in GRO J1655-40, the absorption systems would go in and out of the line of sight and cause temporal variations in the absorption features, which was not observed in our XMMNewton observations.

Acknowledgements. The XMM-Newton project is an ESA Science Mission with instruments and contributions directly funded by ESA Member States and the USA (NASA). The XMM-Newton project is supported by the
Bundesministerium für Wirtschaft und Technologie/Deutsches Zentrum für Luft- und Raumfahrt (BMWI/DLR, FKZ 50 OX 0001), the Max-Planck Society, and the Heidenhain-Stiftung. We acknowledge the RXTE/ASM team for making quick-look results available for public use. We thank Norbert Schulz for pointing out the interstellar origin of the Fe L edges. We acknowledge Marcus Kirsch, Michael Freyberg, Konrad Dennerl, and Cor de Vries for their help with EPIC-pn and RGS calibration issues. We also thank Y. Ueda for useful discussions. G.S. is supported through a postdoctoral fellowship from the Spanish Ministry for Education and Science. X.L.Z. is supported through DLR/FKZ 50 OX 0502.

Note added in proof. Within a few days of placing the refereed accepted version of this paper on astro-ph, two other papers appeared about the same source GRO J1655-40, which partially affect our conclusions and are commented on below.

1. Foellmi et al. (2006) have recently obtained a new determination for the distance to GRO J1655-40 and find an upper limit of $1.7 \mathrm{kpc}$. In this case, the luminosity in the range $0.4-$ $10 \mathrm{keV}$ listed in Table 2 would be $1.0 \times 10^{35}, 9.6 \times 10^{36}, 9.6 \times$ $10^{36}$, and $1.2 \times 10^{37} \mathrm{erg} \mathrm{s}^{-1}$ (for days $27 \mathrm{Feb}$., 14, 15, and 16 March respectively), and the disk inner radius would be only $7.6 \mathrm{~km}, 7.0 \mathrm{~km}$, and $7.2 \mathrm{~km}$ (for days 14, 15, and 16 March 2005). We note, however, that these values are lower than the gravitational radius of the black hole, $R_{\mathrm{g}}=G M / c^{2} \sim 10 \mathrm{~km}$. Therefore, either the distance to GRO J1655-40 is at least $2.5 \mathrm{kpc}$ or the model for deriving the inner radius of the accretion disk requires improved modifications.

2. Miller et al. (2006) reports on a Chandra observation of GRO J1655-40 performed on 1 April 2005, two weeks after our last XMM-Newton observation. The HETGS spectrum shows 90 absorption lines significant at the $5 \sigma$ level of confidence or higher. From all of these, only the Fe XXV K $\alpha$ and $\mathrm{K} \beta$ lines are also present in our March observations. We computed the upper limit for the flux of some of the strongest lines detected by Chandra in the RGS energy range, in particular, for the Fe XXIV 5p, 4p, and 3p lines at 7.159, 7.979, 10.604 , and $10.649 \AA$. Fixing the energy and FWHM of the lines to the values obtained by Miller et al. (2006), we obtain the following upper limits at $3 \sigma$ confidence level for the flux of the lines: $4.2,0.36,1.4$, and $1.3\left(\times 10^{-3} \mathrm{ph} \mathrm{cm}^{-2} \mathrm{~s}^{-1}\right)$, for the observation on 15 March 2005. These upper limits are between a factor 3 and 30 smaller than the fluxes measured in the Chandra spectrum and suggests a change in the ionization state between the two observation periods. 


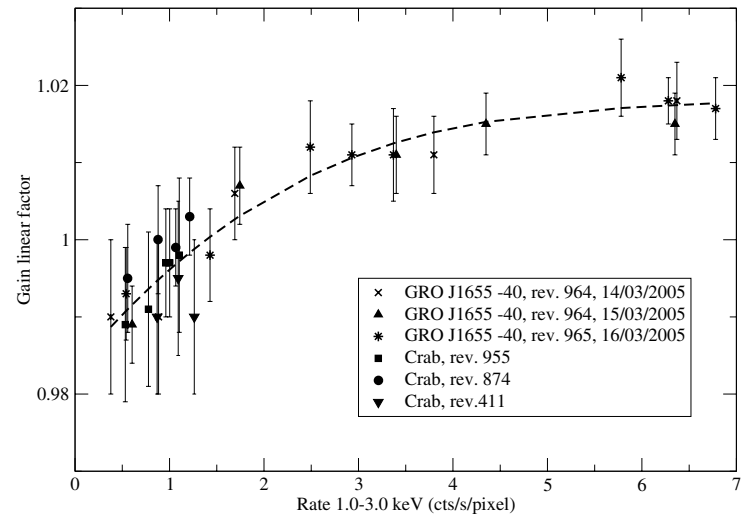

Fig. 7. Calibration of the gain linear factor $f$ as a function of rate per pixel $r$.

\section{Appendix A: EPIC-pn CTE correction}

An inaccurate calibration of the EPIC-pn charge transfer efficiency (CTE) leads to a slightly wrong energy determination of a detected photon, which becomes evident with the large residuals around sharp features like the instrumental Si and Au edges. From our GRO J1655-40 observations, the shift in energy has been found to be rate dependent. Since it is stronger at the center of the PSF, it cannot be directly corrected in the extracted spectrum. We determined the improvement of the CTE (formally handled as a linear gain factor) for different rates, selecting and evaluating the energy-gain linear factor for spectra extracted from different regions of the detector (Fig. 7). Crab calibration observations in the burst mode were also included to improve the determination of the dependence of the gain linear factor $f$ with the rate per pixel $r$ (cts/s/pixel), which we find can be approximated by $f=0.98+0.015 r-2.2 \times 10^{-3} r^{2}+1.1 \times 10^{-4} r^{3}$.

We used this linear gain to correct the energy of our event files: for each photon, the PI channel was divided by the gain factor $f$ according to its rate $r$, assigned to each photon depending on the RAWX column. To avoid the discretization resulting from the pixelization of the PSF in the RAWX columns, the $r$ values were randomized within each RAWX column.

After correcting the event tables with this linear gain, no more residuals appear around the $\mathrm{Au}$ and $\mathrm{Si}$ edges in the new extracted spectra. We confirmed that the correction is also valid in the high energy range, by checking that, after the correction, the spectral features at 7-8 keV have the same central energy both at the center of the PSF (at high rate) and at the wings (low rate).

\section{References}

Balucinska-Church, M., \& Church, M. J. 2000, MNRAS, 312, L55 Brocksopp, C., McGowan, K. E., Krimm, H., et al. 2006, MNRAS, 365, 1203 Costantini, E., Freyberg, M. J., \& Predehl, P. 2005, A\&A, 444, 187 de Vries, C. P., den Herder, J. W., Kaastra, J. S., et al. 2003, A\&A, 404, 959 Dere, K. P., Landi, E., Mason, H. E., Monsignori Fossi, B. C., \& Young, P. R. 1997, A\&AS, 125, 149

Fang, T., Sembach, K. R., \& Canizares, C. R. 2003, ApJ, 586, L49

Foellmi, C., Depagne, E., Dall, T. H., \& Mirabel, F. 2006, A\&A, 457, 249

Greiner, J., Predehl, P., \& Pohl, M. 1995, A\&A, 297, L67

Greiner, J., Cuby, J. G., \& McCaughrean, M. J. 2001, Nature, 414, 522

Harmon, B. A., McCollough, M. L., Zhang, S. N., Paciesas, W. S., \& Wilson, C. A. 1995, IAUC, 6196

Hjellming, R. M., \& Rupen, M. P. 1995, Nature, 375, 464

Homan, J. 2005, ATel, 440

Homan, J., Miller, J. M., Wijnands, R., \& Lewin, W. H. G. 2005, ATel 487

Israelian, G., Rebolo, R., Basri, G., Casares, J., \& Martín, E. L. 1999, Nature, 401,142

Jahoda, K., Markwardt, C., Radeva, Y., et al. 2006, ApJS, 163, 401

Juett, A. M., Schulz, N. S., \& Chakrabarty, D. 2004, ApJ, 612, 308

Juett, A. M., Schulz, N. S., Chakrabarty, D., \& Gorczyca, T. W. 2006, ApJ, 648, 1066

Kallman, T., Liedahl, D., Osterheld, A., Goldstein, W., \& Kahn, S. 1996, ApJ, 465,994

Kotani, T., Ebisawa, K., Dotani, T., et al. 2000, ApJ, 539, 413

Kuster, M., Benlloch, S., Kendziorra, E., \& Briel, U. G. 1999, SPIE, 3765, 673

Landi, E., Del Zanna, G., Young, P. R., et al. 2006, ApJS, 162, 261

Makishima, K., Maejima, Y., Mitsuda, K., et al. 1986, ApJ, 308, 635

Markwardt, C. B., \& Swank, J. H. 2005, ATel, 414

McClintock, J. E., \& Remillard, R. A. 2006, Black Hole Binaries, in Compact Stellar X-ray Sources, ed. W. Lewin, \& M. van der Klis (Cambridge University Press)

Miller, J., Raymond, J., Fabian, A., et al. 2006, Nature, 441, 953

Mirabel, F., \& Rodriguez, L. F. 1994, Nature, 371, 46

Mirabel, F., Rodriguez, L. F., Cordier, B., Paul, J., \& Lebrun, F. 1992, Nature, 358,215

Mirabel, F., Mignani, R., Rodriguez, I., et al. 2002, A\&A, 395, 595

Orosz, J. A., \& Bailyn, C. D. 1997, ApJ, 477, 876

Paerels, F., Brinkmann, A. C., van der Meer, R. L. J., et al. 2001, ApJ, 546, 338

Sala, G., \& Greiner, J. 2006, ATel, 791

Schulz, N., Cui, W., Canizares, C. R., et al. 2002, ApJ, 565, 1141

Sobczak, G. J., McClintock, J. E., Remillard, R. A., Bailyn, C. D., \& Orosy, J. A. 1999, ApJ, 520, 776

Swank, J. H., \& Markwardt, C. B. 2005, ATel, 436

Tingay, S. J., Jauncey, D. L., Preston, R. A., et al. 1995, Nature, 374, 141

Ueda, Y., Inoue, H., Tanaka, Y., et al. 1998, ApJ, 492, 782

van der Hooft, E., Heemskerk, M. H. M., Alberts, F., \& van Paradijs, J. 1998, A\&A, 329, 538

Verner, D. A., Ferland, G. J., Korista, K. T., \& Yakovlev, D. G. 1996, ApJ, 465, 487

Wang, Q. D., Yao, Y., Tripp, T. M., et al. 2005, ApJ, 635, 386

Wilms, J., Allen, A., \& McCray, R. 2000, ApJ, 542, 914

Wilms, J., Juett, A. M., Schulz, N., \& Nowak, M. A. 2006, in preparation

Yamaoka, K., Ueda, Y., Inoue, H., et al. 2001, PASJ, 53, 179

Yao, Y., \& Wang, Q. D. 2005, ApJ, 624, 751

Zhang, S. N., Wilson, C. A., Harmon, B. A., et al. 1994, IAUC, 6046

Zhang, S. N., Cui, W., \& Chen, W. 1997, ApJ, 482, L155 